\title{
TWO-PARTICLE CONTINUOUS-TIME RANDOM WALKS AND BINARY REACTIONS IN DISORDERED MEDIA
}

\author{
R. PARSON and R. KOPELMAN \\ Department of Chemistry, University of Michigan, Ann Arbor, Michigan 48109. USA
}

Received 1 November 1983

\begin{abstract}
The relation between unary (trapping) and binary (mutual annihilation) reactions in disordered systems is studied in the framework of the continuous-time random walk. It is found that if the waiting-time distribution of the walk has infinite moments, a time-independent binary rate constant may exist even though a unary one does not.
\end{abstract}

In recent years, much attention has been devoted to the theory of stochastic transport in disordered systems [1-3]. Most of this work has considered either pure transport properties, such as the meansquared displacement, or the trapping of moving particles at stationary acceptor sites. Less consideration has been devoted to binary reactions between the moving particles, as it is usually assumed that this process is identical with trapping up to a proportionality constant. In the present note, we show that a popular model of transport in disordered systems, the Montroll-Weiss-Scher continuous-time random walk $[4,5]$, gives quite different results for unary and binary reaction rate constants when the waiting-time distribution of the walk has infinite temporal moments. This result is significant because infinite moments are needed to describe non-diffusive behavior such as dispersive transport in semiconductors [5] and fractal transport on percolating clusters [6]. We de not claim that our explicit result holds for such systems, but we do believe that it implies that a simple proportionality between unary and binary rate constants should not be taken for granted in such cases.

We consider a disordered lattice containing two random walkers which destroy each other on contact but otherwise move independently of one another. The precise form of the random motion and the type of disorder are not important for our purposes; for definiteness we may assume that the individual (oneparticle) site occupation probabilites obey a master equation. We define a pair conditional probability or two-particle propagator $P\left(r_{1} r_{2}, t \mid r_{1}^{\prime} r_{2}^{\prime}, t^{\prime}\right)$, the probability that, in the absence of reaction, particle 1 occupies site $\boldsymbol{r}_{1}$ and particle 2 site $\boldsymbol{r}_{2}$ at time $t$ given that they occupied sites $r_{1}^{\prime}$ and $r_{2}^{\prime}$ at time $t^{\prime}$. In each configuration of the disordered system this is a product of one-particle conditional probabilities $p\left(r, t \mid r^{\prime}, t^{\prime}\right)$. The configuration averaged pair probability will not take this form, as the averaging induces an effective interaction between the particles. Our first approximation is to neglect this fact entirely.

If in each configuration the one-particle probabilities obey a master equation, the configuration averaged probability obeys a generalized master equation or equivalently a continuous-time random walk (CTRW) equation [7]. In general, the kernel of the GME or CTRW will have highly non-local spatial properties [8]: we will ignore this and assume that a site-local waiting time distribution is adeguate. We will not 2 ttempt to calculate this function, but merely deduce the twoparticle properties associated with a given waiting-time distribution. This approach is analogous to the very successful Scher-Montroll theory of dispersive transport in amorphous semiconductors [5]. We will consider only qualitative, asymptotic $(r, t \rightarrow \infty)$ properties. It will be seen that even with all of these scvere approximations, we find interesting dynamic behavior.

After configuration averaging (including an average over the initial site [9]) the probabilities become translation-invariant. If all many-body effects are negleeted,

0 009-2614/84/\$ 03.00 @ Elsevier Science Publishers B.V. (North-Holland Physics Publishing Division) 
the incorporation of on-site particle annihilation into the equations of motion becomes a straightforward exercise in Green function and $t$-matrix techniques. The details have been presented by various authors $[10,11]$ and we shall not repeat them; suffice it to say that translation invariance enables one to project the problem onto a trapping one which is then solved by local defect methods. One finds an expression for the steady-state rate constant having the form:

$K^{\prime}(u)=\int_{0}^{\infty} \mathrm{d} t \mathrm{e}^{-u t} K^{\prime}(t)=1 / S(u)$,

$-(\mathrm{d} n / \mathrm{d} t)_{\mathrm{r} \Omega \mathrm{n}}=n^{2} \int_{0}^{t} \mathrm{~d} t^{\prime} K^{\prime}\left(t^{\prime}\right)$,

$S(t)=\sum_{r}\left\langle P\left(r r, t \mid r^{\prime} r^{\prime}\right)\right\rangle$,

in which $n$ is the spatially averaged particle density, held constant in this definition of the steady state, and we assume that at time zero the density was uniform in space. The integral in eq. (2) defines a timedependent rate coefficient; the usual time-independent rate constant is then given by $K^{\prime}(u=0)$. The memory function $S(t)$ is analogous to the diagonal Green function $g(t)=p(r, t \mid r)$ which appears in expressions for trapping rates. Indeed, one may obtain (1) from a first collision time, analogous to a first passage time, definition of the reaction rate. One typically applies (1)-(3) to non-steady-state situations by replacing, in a mean-field approximation, $n$ by $n(t)$. From recent analyses of trapping by dilute acceptors on perfect lattices [12], we know that this approximation will fail at long times; since we expect this problem to be worse in a highly disordered structure we will consider only the steady-state case.

Clearly, the relation between one- and two-particle reaction rates is implicit in that between $g$ and $S$. Let us first assume that all spatial and temporal moments of the CTRW exist. The central limit theorem then yields [4]:

$$
\begin{aligned}
& \left\langle p\left(r, t \mid r^{\prime}\right)\right\rangle \sim(4 \pi D t)^{-d / 2} \mathrm{e}^{-r^{2} / 4 D t}, \quad r, t \rightarrow \infty: \\
& \left\langle p_{k}(t)\right\rangle=\sum_{r} \mathrm{e}^{-\mathrm{i} k \cdot r}\langle p(r, t \mid 0)\rangle \sim \mathrm{e}^{-k^{2} D t}, \\
& \quad k \rightarrow 0, t \rightarrow \infty,
\end{aligned}
$$

in $d$ spatial dimensions, from which we immediately recover the well-known result that the two-particle quantitities are obtained from the one-particle ones by the replacement $D \rightarrow 2 D$. This conclusion also applies to master equations on ordered lattices at all $r$ and $t$, and to CTRWs having infinite spatial but finite temporal moments such as Levy flights [13].

Now let us consider infinite temporal moments. Following Scher and Montroll [5], we classify the asymptotics of the walk according to the long-time decay of the waiting time distribution $\psi(t)$ :

$$
\begin{aligned}
& \psi(t) \sim t^{-(1+\alpha)}, \quad \psi(u) \sim 1-c u^{\alpha} . \\
& 0<\alpha<1 ; \\
& u p_{k}(u)=[1-\psi(u)] /\left[1-f_{k} \psi(u)\right] \\
& \sim u^{\alpha} /\left(u^{\alpha}+\bar{D} k^{2}\right), \quad k, u \rightarrow 0 .
\end{aligned}
$$

In eq. (7), $f_{k}$ is the spatial part of the structure function of the CTRW [4], and $\bar{D}$ is a pseudo-diffusion constant (the mean-squared displacement is sublinear in time) relating length and time seales. Irtegrating (7) over $k$, we find:

$$
\begin{array}{rlrl}
g(t) & \sim t^{-\alpha}, & & d>2 . \\
& \sim t^{-\alpha} \ln t, & d=2, \\
& \sim t^{-\alpha d / 2}, & d<2 .
\end{array}
$$

Eq. (8) suggests that $d=2$ be regarded as an upper critical dimension for CTRWs, above which the characteristic exponents are independent of $d$. For $\alpha=1$, (8) described classical diffusive motion.

To find the asymptotic form of $S(t)$, we proceed in a highly non-rigorous way. We write:

$$
\begin{gathered}
S(t) \sim \int d k k^{d-1}\left[p_{k}(s)\right\}^{2} \\
+ \text { non-singulat part }
\end{gathered}
$$

and use the asymptotic form of $p_{k}(t)$, obtained for $k \neq 0$ from a tauberian theorem:

$p_{k}(t) \sim(1-\alpha) t^{-\alpha} / \bar{D} k^{2} \Gamma(2-\alpha)$

giving:

$S(t) \sim t^{-2 \alpha} \int \mathrm{d} k k^{d-1} /\left(\bar{D} k^{2}\right)^{2}$

which diverges for $d \leqslant 4$, showing that our procedure is indeed faulty: in $d \leqslant 4$ there are important contributions to $(9)$ from $k \approx 0$. To recover sensible results. we absorb the divergence into the time behavior by a 
simple scaling argument. We introduce a lower cutoff $1 / L_{0}$ into (11), and evaluate $S\left(t ; L_{0}\right)$. We then associate a characteristic time $T_{0}$ with $L_{0}$; the form of (7) implies that this should be given by $T_{0}=\left(\bar{D} / L_{0}^{2}\right)^{-1 / \alpha}$. If we now set $T_{0}=t$ we find:

$$
\begin{aligned}
S(t) & \sim \bar{D}^{-2} t^{-2 \alpha}, & & d>4, \\
& \sim \bar{D}^{-2} t^{-2 \alpha} \ln t, & & d=4 \\
& \sim\left[\bar{D}^{-d / 2} /(4-d)\right] t^{-\alpha d / 2}, & & d<4 .
\end{aligned}
$$

We have checked this procedure by applying it to $g(t)$, for which we know the correct result.

Comparison of (12) with (8) shows that the upper critical dimension of the binary reaction is 4 . Alternatively, the effective value of $\alpha$ for the two-particle CTRW is doubled. It follows that for $\alpha>1 / 2, S(t)$ is integrable in dimensions $d>2 / \alpha$. It is thus possible for the binary reaction to possess a non-zero time-independent rate constant $K^{\prime}(u=0)$ even though the unary reaction does not. In the terminology of De Gennes [11], the motion of individual particles is compact, but that of pairs may be non-compact. This is our principal result.

Because of the many approximations involved in the transition from the random walk of two particles in a disordered medium to independent translationinvariant site-local CTRWs, we cannot assess the importance of our result with respect to other long-time anomalies which may arise from those aspects of the dynamics that we have not considered. We are currently designing simulations to study this point. Also, the physical interpretation of our result is unclear, although mathematically it is trivial enough, reducing in essence to: $\left(t^{-\alpha}\right)^{2}=t^{-2 \alpha}$. We suggest that a systematic treatment of this problem, for example a two-particle version of the self-consistent diagrammatic analysis of GAF [3] or of the coherent medium theory [2] would be of interest, though it is likely to be very complicated. We hope that our result suggests some of the qualitatively new features that might arise from a complete theory, or in experiments [14].

This work was supported in part by NSF Grant \#DMR 8303919.

\section{References}

[1] Symposium on Random Walks and their Applications, J. Stat. Phys. 30 (2) (1983).

[2] M. Las and T. Odagaki, in: Macroscopic properties of disordered media, eds. R. Bumridge, S. Childress and

G. Papanicolsou (Springer, Bérlin, 1983);

1. Webman, Phys. Rev. Letters 47 (1981) 1496;

G. Korzeniewski, R. Friesner and R. Silbey, J. Stat. Phys. 31 (1983) 451;

M. Sahimi, B.D. Hughes, L.E. Scriven and H.T. Davis, J. Chem. Phys. 78 (1983) 6849 .

[3] C.R. Gochanour, H.C. Andersen and M.D. Fayer, J. Chem. Phys. 76 (1979) 4254;

R.F. Loring, H.C. Andersen and M.D. Fayer, 1. Chem. Phys. 76 (1982) 2015; to be published;

R.F. Loring, H.C. Andersen and M.D. Fayer, Phys. Rev. Letters 50 (1983) 1324.

[4] G. Weiss and R. Rubin, Advan. Chem. Phys. 52 (1983) 363;

E. Montroll and B.J. West, in: Studies in statistical mechanics, Vol. 7, eds. E. Montroll and J. Lebowitz (North-Holland, Amsterdam, 1979).

[5] H. Scher and E. Montroll, Phys. Rev. B12 (1975) 2455; E. Montroll and H. Scher, J. Stat. Phys. 9 (1973) 101; A. Blumen, J. Klafter and G. Zumofen, Phys. Rev. B27 (1983) 3429.

[6] Y. Gefen, A. Aharony and S. Alexander, Phys. Rev. Letters 50 (1983) 77;

R. Rammal and G. Toulouse, J. Phys. (Paris) 43 (1982) L-265;

S. Alexander and R. Orbach, J. Phys. (Paris) 43 (1982) L-625.

[7] J. Klafter and R. Silbey, Phys. Rev. Letters 44 (1980) 55; J. Chem. Phys. 72 (1980) 843, 849; 74 (1981) 3510.

[8] H. Scher and M. Lax, Phys. Rev. B7 (1973) 4491; M. Shlesinger, J. Klafter and Y. Wong, J. Stat. Phys. 27 (1982) 499

[9] J. Klafter and R. Silbey, Phil. Mag. B47 (1983) 337.

[10] A. Suna, Phys. Rev. B1 (1970) 1716; V. Kenkre, Phys. Rev. B22 (1980) 2089.

[11] P.G. De Gennes, J. Chem. Phys. 76 (1982) 3316.

[12] P. Grassberger and 1. Procaccia, J. Chem. Phys. 77 (1982) 6281;

R.F. Kayser and X. Hubbard, Phys. Rev. Letters 51 (1983) 79.

[13] B.D. Hughes, M.F. Shlesinger and E. Montroll, J. Stat. Phys. 28 (1982) 111.

[14] P.W. Klymko and R. Kopelman, J. Phys. Chem. (1983), to be published. 